Hydroécol. Appl. (1996) Tome 9 Vol. 1-2, pp. 115-137

\title{
Traceurs organiques dans les suspensions et dans la matière organique dissoute du fleuve Sinnamary-barrage de Petit-Saut (Guyane française) : sources et conditions de milieu
}

Organic tracers in total suspended material and dissolved organic matter in the Sinnamary river at Petit-Saut dam (French Guiana) : sources and environmental conditions

\section{F. Gadel(1), L. Serve(2) et J.-L. Blazi(2)}

(1) Laboratoire de Sédimentologie et Géochimie Marines, Université de Perpignan, 52, avenue de Villeneuve, F-66860 Perpignan Cedex.

(2) Laboratoire de Biologie Physico-Chimique, Université de Perpignan, 52, avenue de Villeneuve, F-66860 Perpignan Cedex.

Résumé. - Au cours d'une campagne de mesures réalisée en novembre 1994, les recherches visaient à reconnaître certains biomarqueurs permettant de préciser l'origine des apports et l'influence des conditions de milieu sur l'état de dégradation de la matière organique dans le domaine fluviatile du Sinnamary fortement soumis à des conditions anoxiques, conséquence de la construction du barrage de Petit-Saut.

Les méthodes d'étude mises en œuvre ont compris l'analyse pondérale de la charge en suspension (matières en suspension: MES), le dosage du carbone organique particulaire (COP) et dissous (COD), des polysaccharides (colorimétrie), et, par des techniques plus fines, l'analyse des composés phénoliques (chromatographie liquide à haute performance: CLHP) et celle de classes principales de composés organiques (pyrolyse-chromatographie gaz-liquide couplée à la spectrométrie de masse: Py-CG-SM). Dans le secteur amont du réservoir, les apports fluviatiles directs sont marqués par des teneurs élevées en hydrocarbures aromatiques et en phénols correspondant à un matériel issu du lessivage des sols. A l'aval du réservoir, les teneurs en matière organique s'accroissent dans le matériel en suspension par suite de la baisse de la charge minérale. Un enrichissement en sucres, notamment en polysaccharides, et en sucres aminés peut être mis en rapport avec le développement du phytoplancton.

A proximité du barrage, sur des profils verticaux, les sucres et les composés azotés, 
contrairement aux phénols résistants, décroissent vers la profondeur en rapport avec le développement du phytoplancton en surface la dégradation de la matière organique et avec l'anoxie des couches profondes. Le plus grand etat de fraîcheur de la matiere organique dissoute se traduit par l'accroissement des sucres aminés et la baisse des hydrocarbures aromatiques.

En aval du barrage, un enrichissement en matière organique se manifeste au niveau de l'estuaire. Les teneurs en phénols et en polysaccharides (complexe ligno-cellulosique) décroissent vers l'aval tandis que s'accroissent les composés azotés, indicateurs d'un caractère marin plus marqué.

Mots-clés. - Fleuve. Guyane française, Sinnamary, matières en suspension (MES), carbone organique particulaire (COP). carbone organique dissous (COD), polysaccharides, composés phénoliques, lignine, classes majeures de composés organiques.

Abstract. - During a measurement survey in November 1994, research focused on identifying certain biomarkers in the Sinnamary river which could point up the origin of inputs and the influence of environmental conditions on the degree of degradation of the organic matter in a fluvial area strongly subjected to anoxic conditions resulting from the construction of the Petit-Saut dam.

The methods adopted included analysis of the suspended load (total suspended matter: TSM), particulate and dissolved organic carbon, polysaccharides, and, by more precise techniques, the analysis of phenolic compounds (HPLC) and other main classes of organic compounds (PY-GCMS).

Upstream, the direct fluvial input is characterized by high concentrations of aromatic hydrocarbons and phenols, corresponding to products released due to soil leaching. Downstream, the increase in carbohydrates, particularly in polysaccharides, and in aminosugars can be related to phytoplankton growth. Organic matter increases in the suspended material due to the decrease of the suspended mineral load.

Along a vertical profile near the dam, sugars and nitrogen compounds decrease with depth, unlike the resistant phenols, as phytoplankton develop on the surface and the deep layers become anoxic. The fresher state of the dissolved organic matter leads to an increase in amino sugars and a drop in aromatic hydrocarbons.

Downstream, the organic matter is enriched near the estuary. Phenol and polysaccharide concentrations (ligno-cellulosic complex) decrease with the distance from the dam, while nitrogen compounds increase, indicating a more marked marine character.

Keywords. - River, French Guiana, Sinnamary, total suspended matter (TSM), particulate organic carbon (POC), dissolved organic carbon (DOC), polysaccharides, phenolic compounds, lignin, main classes of organic compounds. 


\section{INTRODUCTION}

Les fleuves constituent l'élément majeur du transport de la matière de la terre à l'océan (Garrels et al. 1973). Les premières études sur les matières particulaires et dissoutes transportées par les fleuves ont été réalisées par Livingstone (1963) et Alekin (1978). Par la suite de nombreux travaux se sont intéressés à la matière organique particulaire (MOP) et dissoute (MOD) et à la phase minérale des eaux des fleuves : argiles, sels nutritifs, métaux (Martin et Meybeck, 1979).

Poursuivant les travaux déjà réalisés par les laboratoires perpignanais sur le delta du Rhône (Gadel et al., 1992 ) et d'autres fleuves comme le Congo et l'Amazone (Programme PEGI) ou encore la Seine (Programme Piren Seine), nous avons entrepris l'étude des caractères de la matière organique dans un fleuve sud-américain de la zone tropicale, le Sinnamary (Guyane française, fig. 1), caractérisé par la désoxygénation de ses eaux consécutive à la construction du barrage de Petit-Saut et avons tenté de répondre aux questions: Quelles sont les formes chimiques organiques majeures dans les eaux du fleuve? Quelle est leur origine? Quels facteurs environnementaux contrôlent leur distribution?

La matière organique provient de deux sources principales : une origine aquogénique (phytoplancton et plantes aquatiques) et une origine pédogénique (lessivage des sols) (Buffle et al., 1987). Les caractères biogéochimiques initiaux dépendant de l'ori- gine du matériel sont ensuite quelque peu altérés par les conditions de milieu dépendant du $\mathrm{pH}$, du degré d'oxygénation, de la température des eaux, de l'activité bactérienne et d'autres facteurs indirects comme la longueur du cours, la pente du lit, les turbulences et les zones de calme, la proximité de l'embouchure (Meybeck, 1982). Du résultat de l'action de ces divers facteurs résulte une composition variable de la matière organique qui est fonction des sites de prélèvement.

Aussi, le but de la mission qui s'est déroulée en novembre 1994, en liaison avec le Laboratoire d'Hydrobiologie de Petit-Saut, à la demande des Services de I'EDF-CNEH, était de reconnaitre la distribution de la matière organique et ses caractères biogéochimiques dans l'écosystème aquatique constitué par le fleuve et la retenue du barrage de Petit-Saut (fig. 1). En parallèle, ont été réalisées des études sur le phytoplancton et les populations microbiennes (Laboratoire d'Hydrobiologie Marine et Continentale-Montpellier II et Centre d'Océanologie de Marseille).

En ce qui nous concerne, l'étude réalisée sur la matière organique a compris :

1. l'analyse quantitative à partir du carbone organique particulaire (COP) et dissous (COD).

2. l'analyse qualitative de la matière organique dans les MES à partir du dosage des polysaccharides, des composés phénoliques, principalement ceux de la lignine, et de certaines classes majeures de composés organiques. 


\section{MATÉRIEL ET MÉTHODES D'ÉTUDE}

Au cours de la mission, ont été effectués des prélèvements d'eau et les filtrations correspondantes sur filtres Whatman en fibres de verre (GF/F) afin de séparer le matériel en suspension, ainsi que quelques lyophilisations de la matière organique dissoute après filtration des eaux à $0,45 \mu \mathrm{m}$. Des échantillons de végétaux ont été recueillis; les résultats de leur analyse sont seulement évoqués ici.

Les prises d'eau effectuées en surface au cours de la mission correspondent à plusieurs campagnes de prélèvement concernant 15 stations (fig. 1) :

- Descente amont de Saut-Mouche à BPS (9 stations le 17/11/1994),

- Remontée aval d'Estuaire à Dégrad (6 stations le 15/11/1994),

- Prélèvements à diverses profondeurs sur la station BPS (12/11 au 18/11/94).

Les analyses réalisées concernent :

- la charge ou teneur en matières en suspension (MES, obtenue par filtration sur filtres GF/F),

- le carbone organique particulaire et dissous,

- les polysaccharides,

- les composés phénoliques,

- certaines classes majeures de composés organiques.

\subsection{Analyse quantitative de la ma- tière organique}

L'analyse du carbone organique particulaire (COP, exprimé en pourcents de la prise initiale) est réalisée par combustion sèche dans un four à induction sous courant d'oxygène avec mesure par infra-rouge du dioxyde de carbone formé sur un analyseur LECO CS 125 après décarbonatation de l'échantillon.

L'analyse du carbone total, obtenue sans décarbonatation, permet alors d'évaluer les teneurs en carbone minéral par soustraction du carbone organique: Ctotal $-\mathrm{COP}=$ Cminéral. L'analyse du carbone organique dissous (COD) est effectuée sur les prises d'eau initiales avec un appareil Shimadzu T 4000.

\subsection{Analyse qualitative de la ma- tière organique}

L'analyse globale des polysaccharides est réalisée par colorimétrie avec le réactif anthrone (Gallali, 1972). Les valeurs sont exprimées en $\mathrm{mg} / \mathrm{g}$, en $\mathrm{mg} / \mathrm{l}$ et rapportées au COP.

L'analyse des composés phénoliques, produits du métabolisme secondaire des végétaux supérieurs et contenus dans les restes ligneux ou les colloïdes du sol, s'effectue, après oxydation alcaline au $\mathrm{CuO}$ et extraction liquide-liquide par chromatographie liquide à haute performance (CLHP) avec un appareil Shimadzu LC-6A (Charrière et al., 1986). Les composés sont identifiés par comparaison avec des produits de référence. 

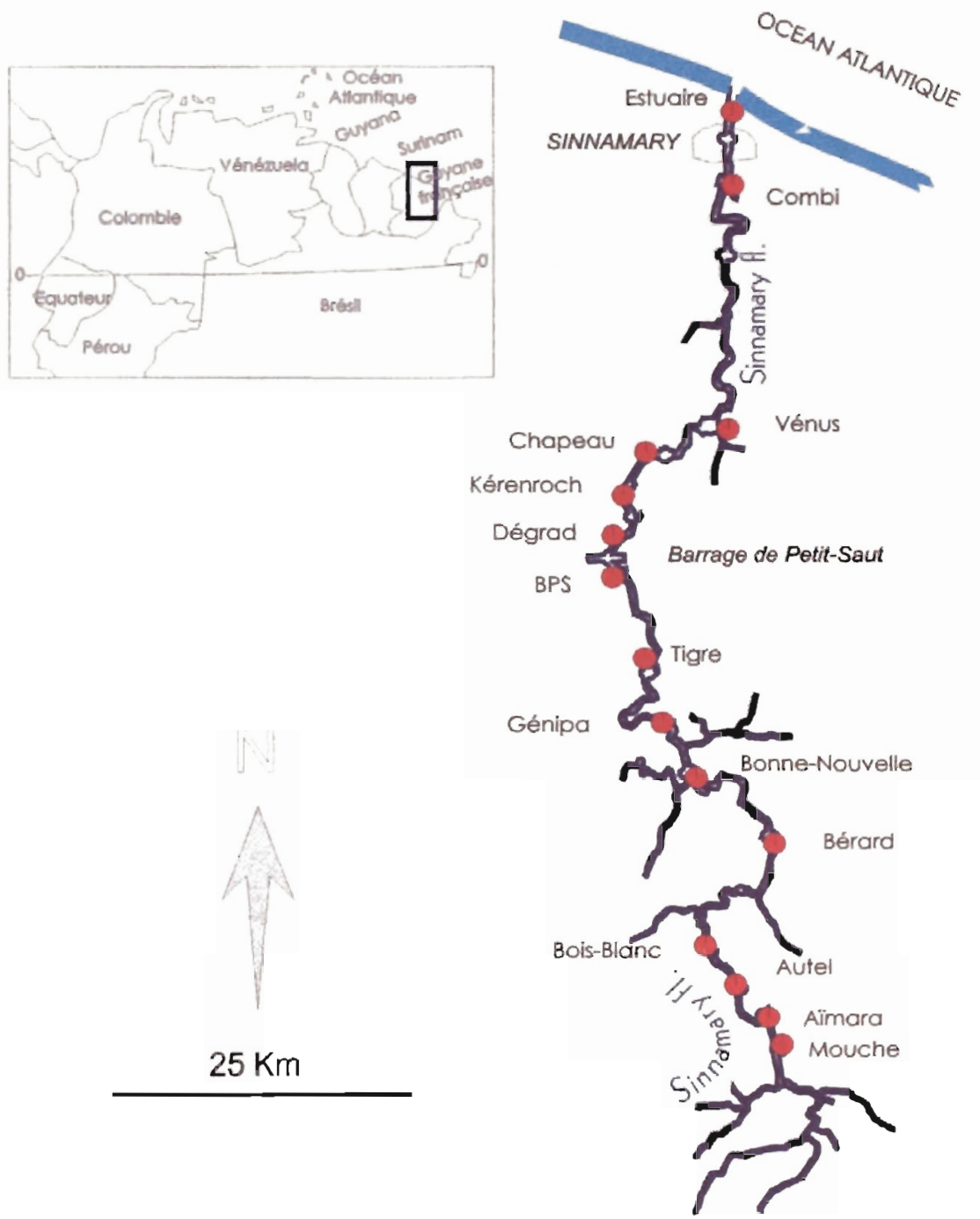

Fig. 1. - Vue gènérale et situation des stations d'éfude sur le fleuve Sinnamary.

Fig. 1. - General overview and location of sampling stations along the Sinnamary River. 
Deux standards internes (phloroglucinol et acide $p$-anisique) sont utilisés pour les évaluations quantitatives. Un gradient complexe de haute pression est utilisé avec deux éluents ternaires composés d'eau, d'acétonitrile et d'acide acétique. Les teneurs sont exprimées en $\mathrm{mg} / \mathrm{g}$, et rapportées au COP.

L'analyse des classes majeures de composés organiques: hydrocarbures aromatiques, composés azotés, sucres, composés phénoliques et sucres aminés est effectuée par un couplage pyrolyse-chromatographie gaz-liquidespectrométrie de masse à l'Institut Químic de Sarrià de Barcelone.

Une sonde de pyrolyse C.D.S. 1000 est couplée directement à un chromatographe en phase gazeuse PerkinElmer 8700 équipé d'une colonne capillaire à phase stationnaire polaire constituée de silice fondue greffée avec du polyéthylèneglycol (Supelcowax 10) de $30 \mathrm{~m}$ de longueur, de $0,25 \mathrm{~mm}$ de diamètre interne et dont la phase mesure $0,25 \mu \mathrm{m}$ d'épaisseur. La température de pyrolyse est de $700{ }^{\circ} \mathrm{C}$ durant $10 \mathrm{~s}$. La température de la colonne est programmée de $50^{\circ} \mathrm{C}$ jusqu'à $240^{\circ} \mathrm{C}$ suivant un gradient de $6^{\circ} \mathrm{C}$ par minute. Les fragments de pyrolyse sont identifiés par un couplage avec un spectrometre de masse HP 5989. Les pyrochromatogrammes très complexes permettent d'isoler 23 pics majeurs dont la somme des aires est rapportée à 100\%. Chacun des composés sélectionnés correspond alors à un certain pourcentage défini de cette aire. Les fragments de pyrolyse analysés sont donc exprimés en pourcentages relatifs.

\section{RÉSULTATS}

\subsection{Analyse de la matière en sus- pension, du carbone organique particulaire et dissous}

\subsubsection{Secteur amont du barrage, de Saut-Mouche à BPS}

La charge (MES) en mg/l décroît en surface de la station Mouche à la station Bérard, le jour du prélèvement (17/11/94) et s'accroît ensuite vers l'aval jusqu'à BPS, soulignant l'incidence des apports fluviatiles tout-à-fait en amont et du développement du phytoplancton vers l'aval (fig. 2).

Les teneurs en carbone organique particulaire (mg/l) décroissent jusqu'à Bois-Blanc et s'accroissent ensuite jusqu'à BPS (fig. 2), ce qui peut être rapporté au développement du phytoplancton souligné par la distribution de la chlorophylle a (A. Vaquer, 1997). Les valeurs en pourcents, assez faibles en amont jusqu'à BoisBlanc (dilution par la charge minérale remise en suspension), s'accroissent ensuite fortement (faible charge minérale).

Les teneurs en carbone minéral particulaire sont très faibles (moyenne: $1,5 \%$ ), ce qui peut s'expliquer par l'acidité des eaux en rapport avec un substratum granitique et des séries métamorphiques siliceuses. Dans la partie moyenne, elles peuvent atteindre 2 à $4 \%$ à Aïmara, Autel, Bois-Blanc, peut-être en rapport avec la présence de microorganismes a test carbonaté. 
¿

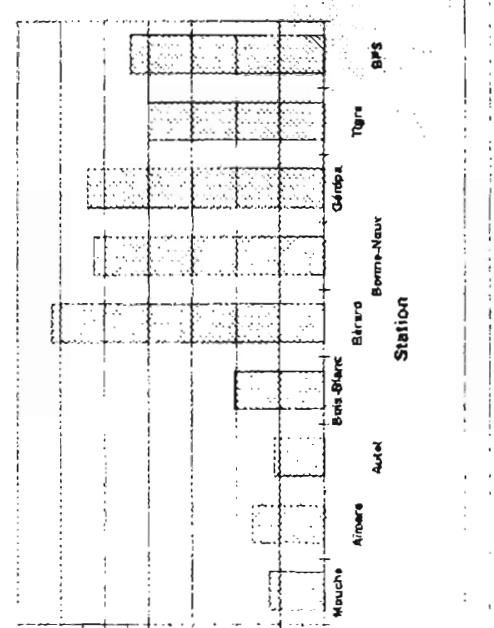

요 के ร \% 2 웅

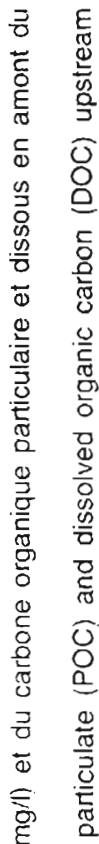

$\underset{\Sigma}{\stackrel{\oplus}{\infty}}$

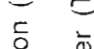

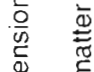

Q ह के Q

ब) $\dot{0} \frac{\bar{\Delta}}{\overline{0}} \dot{0}$ 可 $\frac{\pi}{\bar{c}} \bar{c} \frac{c}{\omega}$ 뜨을 $\frac{0}{\partial} \overline{0} \div$ () 离产要 문 व 8

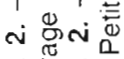

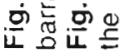

Les teneurs en carbone organique dissous évoluent de 3 à $7 \mathrm{mg} / \mathrm{l}$, mais sur Aimara la valeur est très forte
(52 mg/l), indice possible d'apports latéraux issus du lessivage des sols (fig. 2). Le rapport COD/COP, s'il évo- 
lue de 3 à 10 sur la plupart des stations, s'élève alors fortement sur Aïmara.

\subsubsection{Secteur aval du barrage, de Dégrad à Estuaire}

Dans la joumée du 15/11/94, la charge en mg/l en surface tend à s'accroître vers l'aval, notamment dans l'estuaire en raison des turbulences et des effets de floculation possibles au contact des eaux salées (fig. 3). Comme les prélèvements ont été effectués dans cette zone à $14 \mathrm{~h}$ environ, que les masses d'eau mettent environ deux jours pour franchir la distance du barrage à l'estuaire et que les décharges du barrage se font par ouverture des vannes de fond, l'augmentation de la charge observée dans la partie aval peut être circonstancielle.

Les teneurs en carbone organique particulaire (mg/l) s'accroissent aussi vers l'aval. Les valeurs sont plus fortes ici qu'en amont du barrage, notamment vers l'estuaire (fig. 3). Les teneurs en pourcents tendent au contraire à décroître vers l'aval, certainement par suite de la dilution introduite par une charge minérale plus abondante (remise en suspension). La valeur la plus forte est observée sur Kérenroch.

Les teneurs en COD sont plus élevées en amont avec un maximum sur Kérenroch et décroissent vers l'aval (fig. 3). La distribution des valeurs du rapport COD/COP montre donc une baisse des valeurs vers l'aval en rapport avec la plus grande abondance de la matière organique particulaire et la dégradation de la matière organique dissoute dans les masses d'eau.

Par l'analyse du matériel en suspension on a montré qu'après turbinage les eaux présentaient un baisse des teneurs en COP, en polysaccharides et au contraire un accroissement des phénols, signe d'une accentuation de la dégradation de la matière organique probablement en rapport avec l'origine profonde de ces eaux turbinées.

\subsubsection{Station BPS-Profils verticaux}

La charge (MES) décroît généralement vers la profondeur, excepté le $12 / 11$, car le fond a été touché (remise en suspension) (fig. 4).

La charge organique décroît aussi vers la profondeur: signe de dégradation. II faut noter un acroissement à $3 \mathrm{~m}$ de profondeur le 18/11, certainement consécutif au développement du phytoplancton comme le montrent aussi les teneurs plus fortes en chlorophylle a (A. Vaquer, 1997).

Les teneurs en carbone organique en pourcents décroissent également vers la profondeur, mais s'accroissent au contraire le 18/11 en raison d'une faible charge minérale (fig. 4).

\subsection{Analyse des polysaccharides}

Les teneurs exprimées en $\mathrm{mg} / \mathrm{l}$ sont certainement plus exactes que les valeurs exprimées en $\mathrm{mg} / \mathrm{g}$ ou rapportées au carbone organique particulaire total. En effet, ces dernières valeurs dépendent d'une part de la 

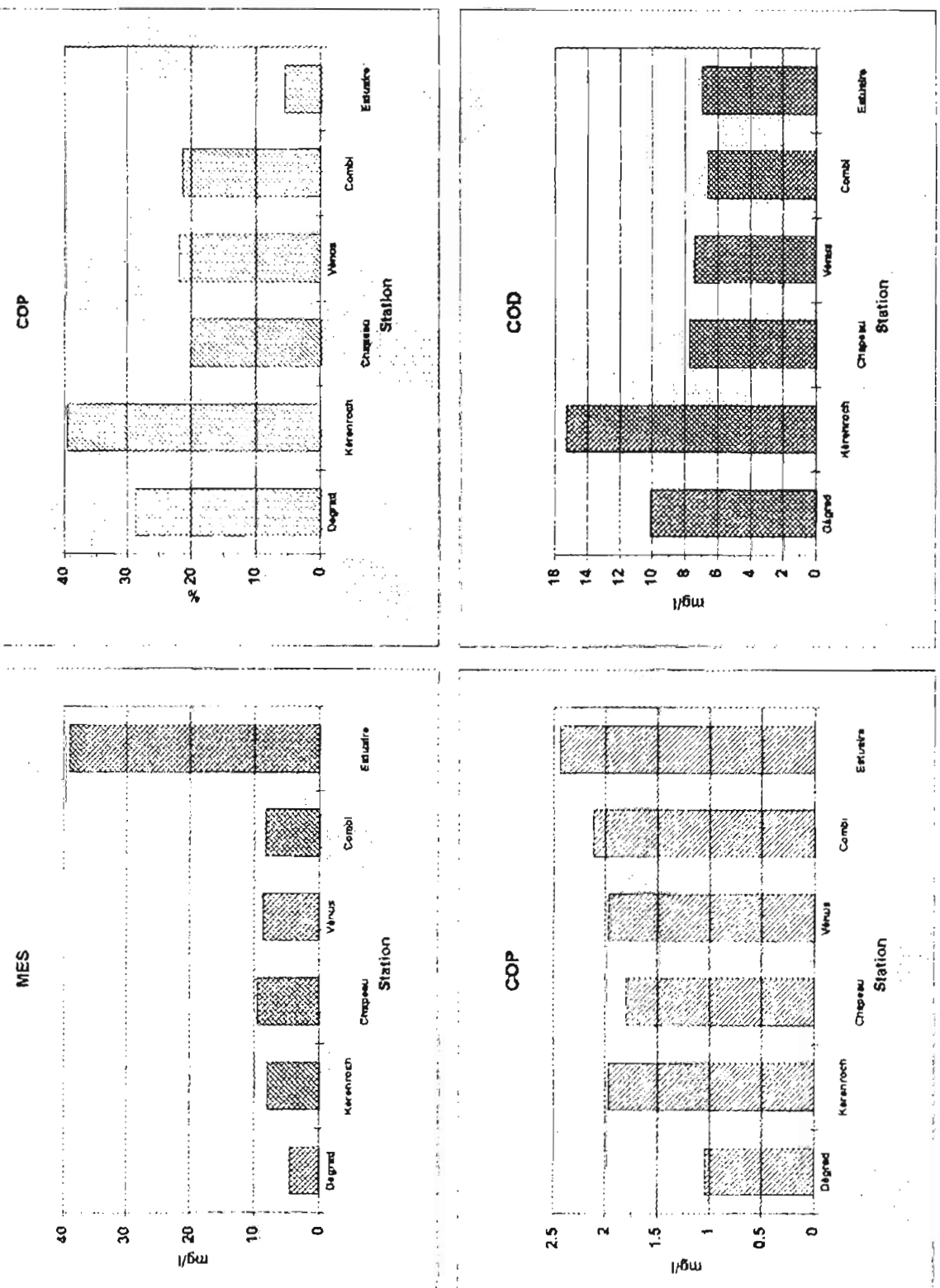

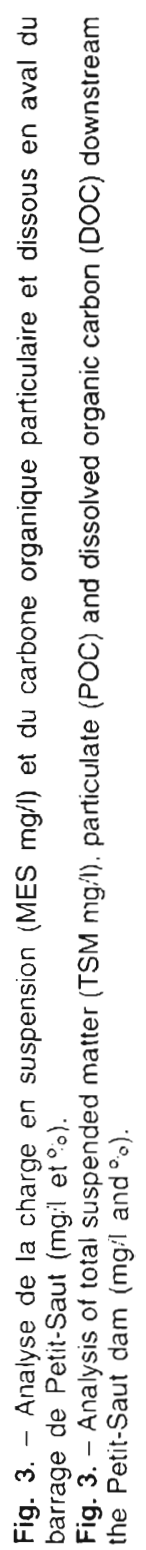



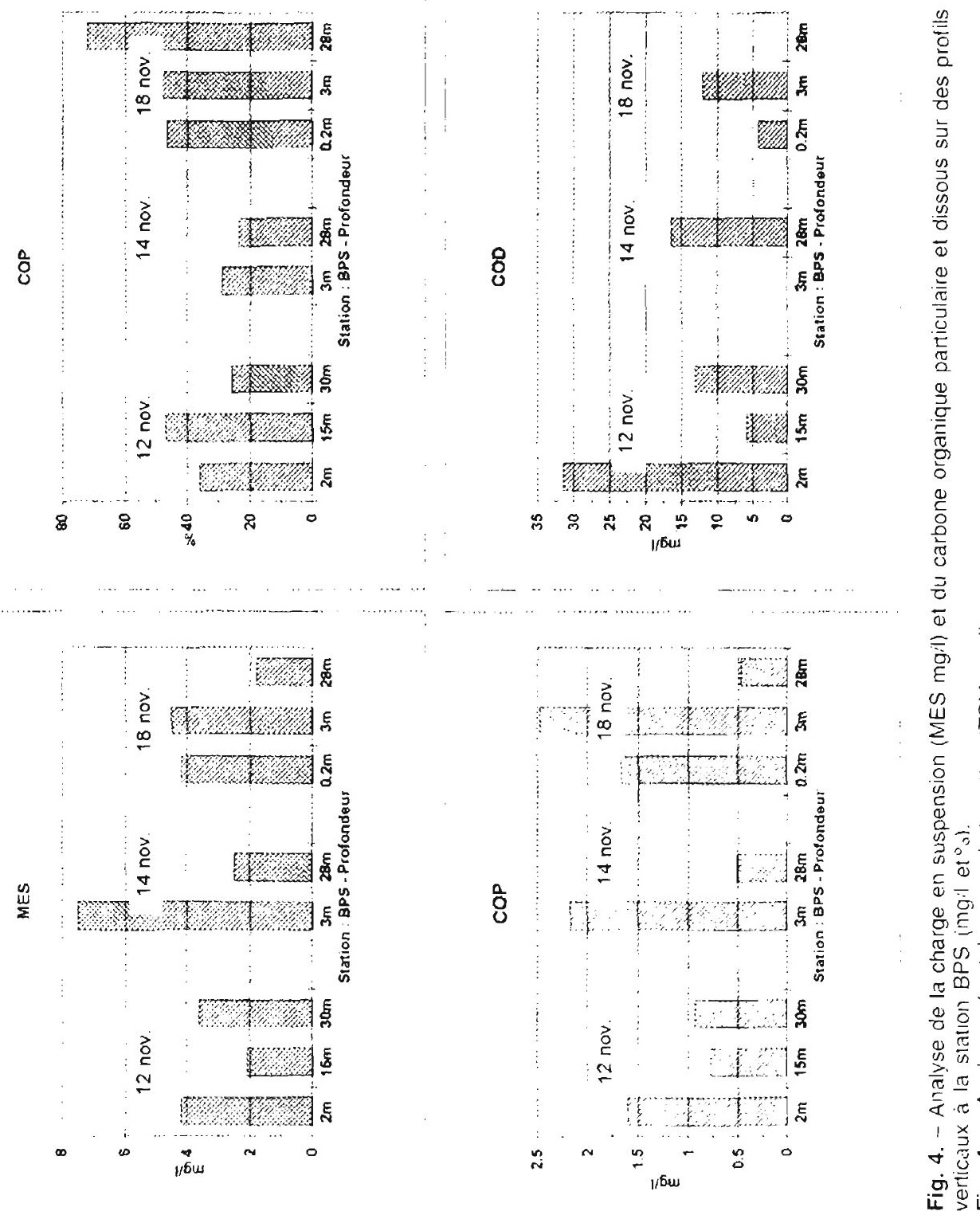
charge totale assez imprécise, car très faible, et d'autre part de la teneur en carbone organique particulaire analysée sur un autre filtre.

\subsubsection{Secteur amont, de Saut- Mouche à BPS}

Les teneurs en $\mathrm{mg} / \mathrm{l}$ sont faibles en amont en surface (prélèvement effectué le 17/11/94) jusqu'à Autel, pour s'accroître fortement à partir de Bérard (fig. 5A). Les valeurs en $\mathrm{mg} / \mathrm{g}$, très faibles en amont jusqu'à Autel (influence des charges minérales fluviatiles issues du lessivage des sols), s'accroissent à partir de Bois-Blanc avec des valeurs maximales sur Bérard et BPS (développement du phytoplancton comme le montre l'accroissement de la chlorophylle a (A. Vaquer, 1997).

Les teneurs exprimées en \% C polysacch./C org. particulaire suivent une évolution assez voisine avec des valeurs très fortes sur Bérard et BPS (fig. 5A).

\subsubsection{Secteur aval, de Dégrad à Estuaire}

Les teneurs en $\mathrm{mg} / \mathrm{l}$ (prélèvement du 15/11/94) sont relativement fortes sur Chapeau, plus basses sur Kérenroch (fig. 5B).

Les teneurs en $\mathrm{mg} / \mathrm{g}$ sont fortes sur Dégrad et décroissent ensuite jusqu'à l'embouchure (accroissement de la charge minérale). Les valeurs exprimées en \% C polysacch. /C org. particulaire sont plus fortes sur Dégrad et Chapeau et décroissent aussi vers l'aval. On note une valeur faible sur Kérenroch (fig. 5B).
Le secteur estuarien paraît moins riche en polysaccharides que le domaine fluviatile franc. Par ailleurs, les teneurs en polysaccharides, généralement plus faibles en aval qu'en amont du barrage, peuvent traduire une dégradation de la matière organique en suspension au cours de son transit dans la masse liquide ou souligner le fait qu'elles sont issues des eaux profondes du barrage riches en matériel dégradé.

\subsubsection{Station BPS-Profils verticaux}

II faut remarquer que, pour les valeurs exprimées en $\mathrm{mg} / \mathrm{l}$, le niveau à $3 \mathrm{~m}$ paraît très riche en sucres le 18/11 (présence de phytoplancton) comme le montrent les valeurs fortes de la chlorophylle a (A. Vaquer, dans ce volume; fig. $5 \mathrm{C}$ ).

Les teneurs en $\mathrm{mg} / \mathrm{g}$ décroissent vers la profondeur, excepté le 14/11, par suite de la plus grande richesse en phytoplancton en surface, des effets de dégradation dans la colonne d'eau et de l'accroissement de la charge minérale en profondeur (fig. $5 \mathrm{C}$ ). L'évolution est sensiblement la même pour les valeurs rapportées au carbone organique particulaire.

\subsection{Analyse des composés phénoliques}

Les composés phénoliques analysés correspondent aux composés polyhydroxyaromatiques dérivés des lignines présentes dans les suspensions et provenant des végétaux supérieurs ainsi que des horizons 

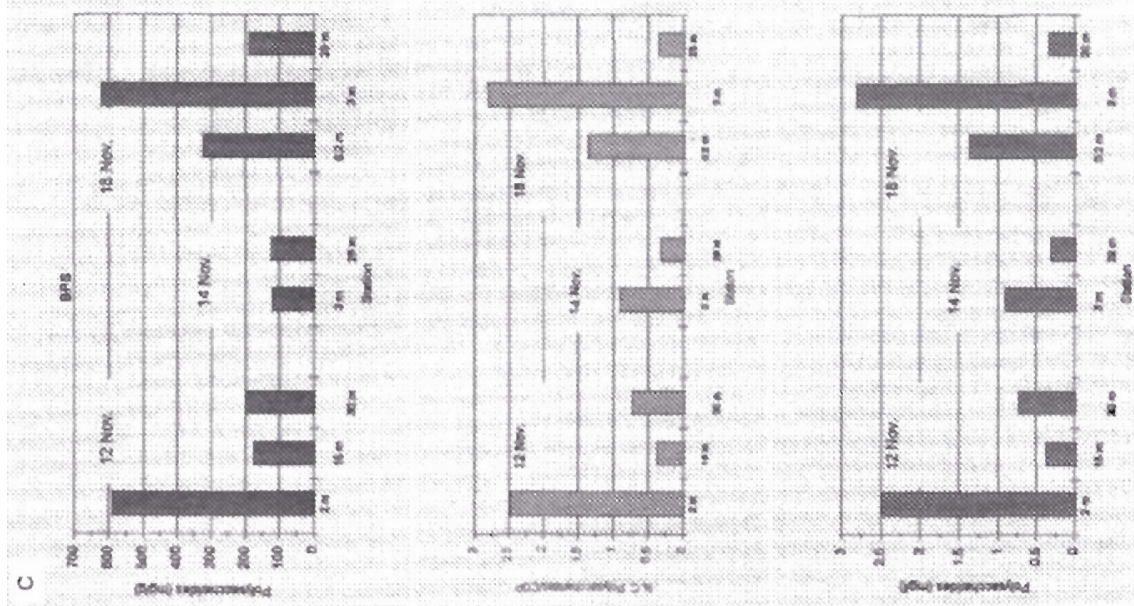

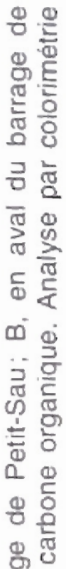
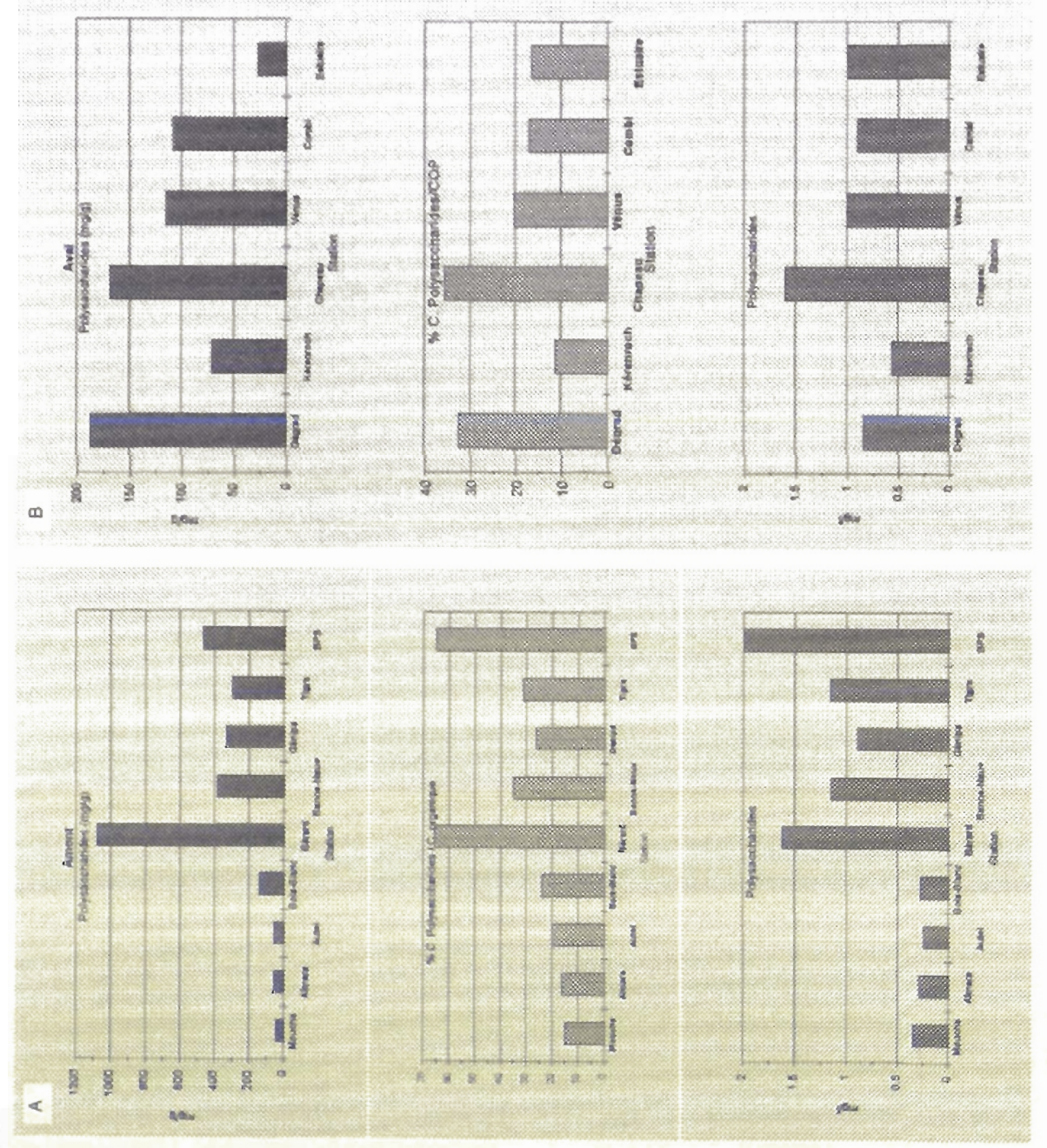
humifères terrestres (Ibrahim et al., 1962 ; Hartley, 1971; Hartley et Buchan, 1979 ; Pocklington et McGregor, 1973; Hedges et Parker, 1976; Hedges et Mann, 1979; Hedges et Ertel, 1982 ; Hedges et al., 1986). Les structures hydroxybenzyliques peuvent, dans certaines conditions, être rapportées pour partie aux organismes phytoplanctoniques.

\subsubsection{Secteur amont, de Saut-Mouche à BPS}

Le fait majeur est l'accroissement des teneurs en $\mathrm{mg} / \mathrm{g}$ remarqué à partir de Bois-Blanc jusqu'en aval (dilution moindre par la charge minérale; fig. 6A). Compte tenu de l'augmentation concomitante des polysaccharides, il semble difficile d'apporter une explication. Toutefois, les phénols peuvent pour certains, comme les hydroxybenzyles, être issus du phytoplancton ou des feuilles, une des sources supposée des polysaccharides. L'analyse du bois et des feuilles de certains arbres de la forêt avoisinante a en effet bien montré que les phénols hydroxybenzyles étaient plus abondants dans les feuilles.

Les teneurs rapportées au carbone organique (à diviser par deux pour obtenir les valeurs rapportées à la matière organique) montrent un accroissement sur Bois-Blanc et Bérard (fig. 6A).

Les apports de matériel ligneux, quantifiés par la somme des composés vanilliques, syringiques et cinnamiques $(\mathrm{V}+\mathrm{S}+\mathrm{C}$; Hedges et Parker, 1979), exprimée en pourcents du carbone organique particulaire, présen- tent des valeurs très fortes sur Bérard, un peu moins sur Mouche et Génipa, faibles enfin sur Aimara, Bois-Blanc, Bonne-Nouvelle et BPS (fig. 6B). Les valeurs de ces mêmes phénols rapportées aux phénols totaux sont fortes sur Mouche et Génipa. Dans le cas de Mouche, cela n'a rien d'étonnant pour un matériel directement issu du lessivage des sols. Sur les autres stations, les valeurs décroissent vers l'aval. La station Bérard présente des valeurs très fortes en hydroxybenzyles, comme Bois-Blanc. A BoisBlanc, les phénols hydroxybenzyles qui prédominent peuvent être d'origine phytoplanctonique ou issus des feuilles (fig. 6C). Sur Bérard, associés à des phénols d'origine ligneuse, s'observent des phénols d'origine phytoplanctonique, mais également des phénols simples et de l'acide benzoïque. Sur Génipa, les phénols d'origine ligneuse prédominent (apport d'un affluent).

Les hydroxybenzyles rapportés aux phénols totaux offrent des valeurs assez peu variables, excepté sur Génipa avec une valeur très faible. Rapportés au carbone organique particulaire un accroissement se manifeste à partir d'Autel jusqu'à Bérard (phytoplancton), avec une décroissance ensuite (fig. 6C).

La distribution des composés phénoliques semble donc assez hétérogène dans ce secteur du fait de la grande diversité des apports : produits issus du lessivage des sols apportés par les affluents ou ceux libérés par le phytoplancton, les plantes aquatiques ou les feuilles. Sur les diverses 
A

Amont
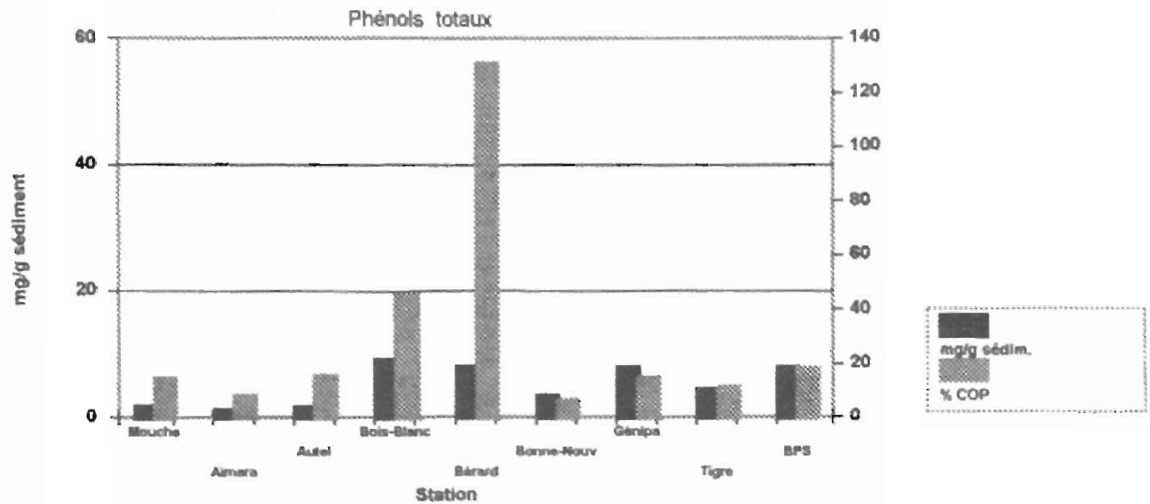

B
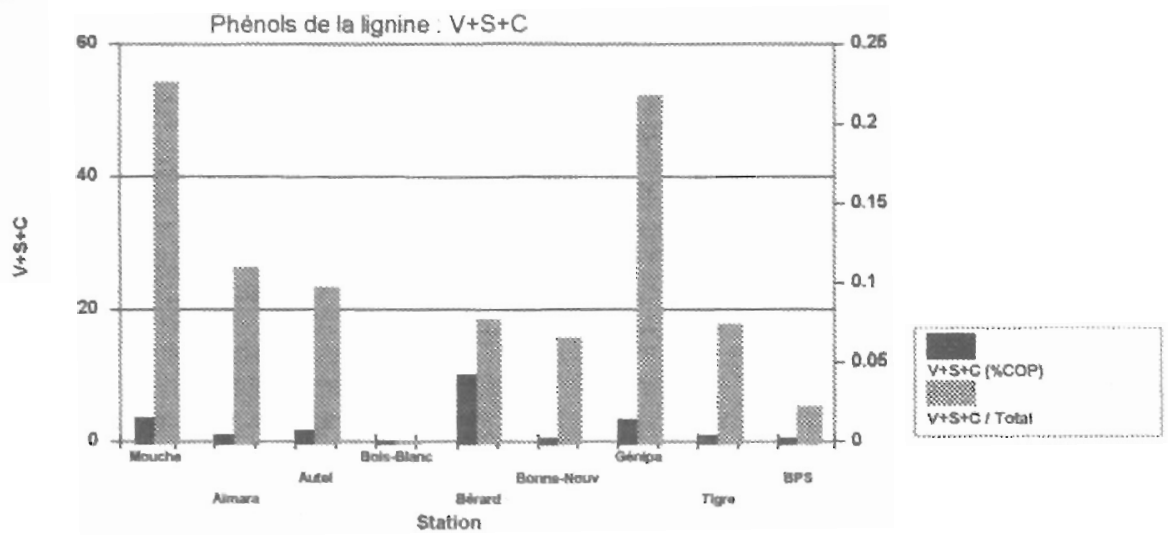

C

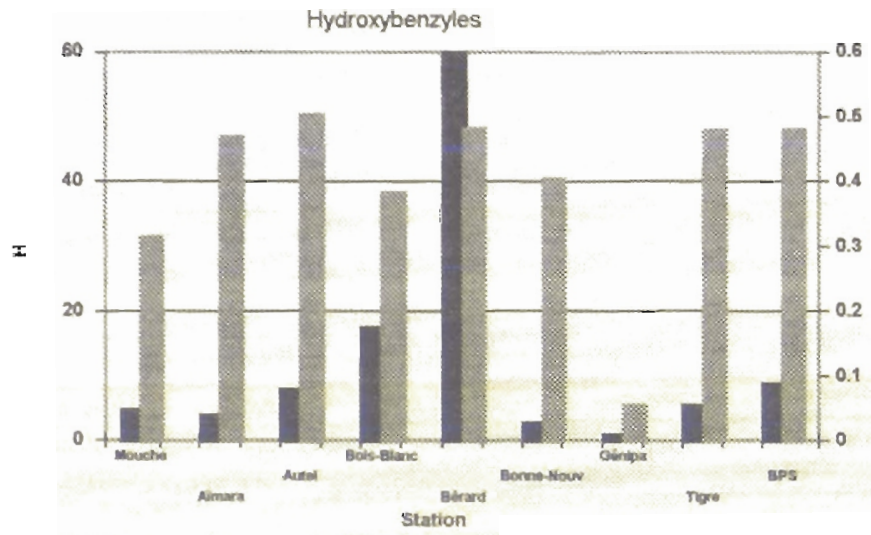

Fìg. 6. - Teneurs en composés phénoliques dans le maiériel en suspension en amont du barrage de Petit-Saut. A : Teneurs totales en phénols $(\mathrm{mg} / \mathrm{g}-\%$ COP), B : composés phénoliques dérivés de la lignine c'est-à-dire Teneurs en vanillyles, syringyles et cinnamyles $(\mathrm{V}+\mathrm{S}+\mathrm{C})(\%$ COP-\% phénols totaux), C: composés hydroxybenzyles $(\%$ COP-\% phénols totaux).

Fig. 6. - Contents in phenolic compounds in total suspended material upstream the Petit-Saut dam. A : Total Phenol contents ( $\mathrm{mg} / \mathrm{g}=\%$ COP), B : Lignin-derived phenolic compounds i.e. Vanillyl, Syringyl and Cinnamyl contents $(\mathrm{V}+\mathrm{S}+\mathrm{C})(\% \mathrm{POG}-\%$ total phenols), C : Hydroxybenzyl compounds (\% POC-\% total phenols). 
stations, on remarque soit une prédominance du matériel ligneux riche en $V+S+C$ (le fleuve ou ses affluents), soit celle de matériel phytoplanctonique aquogène ou de matériel foliaire riche en hydroxybenzyles.

\subsubsection{Secteur aval, de Dégrad à Estuaire}

Les teneurs en phénols $(\mathrm{mg} / \mathrm{g})$, en surface, sont plus régulières qu'en amont et la distribution des phénols rapportés au carbone organique particulaire montre un fort accroissement des valeurs comparativement au secteur amont, excepté sur Kérenroch (fig. 7A). Ce fait peut témoigner d'un état plus dégradé de la matière organique issue des eaux de fond du barrage. La consenvation relative de ces composés résistants s'accompagne au cours de la dégradation d'une baisse des composés biodégradables comme les sucres et certains composés azotés.

La teneur en $\mathrm{V}+\mathrm{S}+\mathrm{C}$ est forte sur Kérenroch et les valeurs excessivement faibles ailleurs (fig. 7B). Les phénols hydroxybenzyles, probablement issus du phytoplancton ou des feuilles, sont bien représentés sur toutes les stations, notamment vers l'aval (fig. 7C).

\subsubsection{Analyse sur BPS - Profils verticaux}

Les phénols sont analysés à la fois sur la matière particulaire et dissoute le 18/11 à plusieurs niveaux de la colonne d'eau (fig. 8A et 8B). Dans la matière organique particulaire, les teneurs rapportées au carbone organique s'accroissent avec la profondeur aux trois dates de prélèvement, témoignant d'une bonne conservation de ces composés résistants. Les teneurs sont plus fortes dans la matière organique dissoute en surface et plus faibles en profondeur (fig. 8B).

Les teneurs en $\mathrm{V}+\mathrm{S}+\mathrm{C}$ rapportées au carbone organique particulaire diminuent vers la profondeur. Rapportées aux phénols totaux, les valeurs s'accroissent au cours de la dégradation, sauf pour les échantillons prélevés le 18/11/94. Les phénols hydroxybenzyles rapportés aux phénols totaux décroissent dans les couches profondes (résistance moindre à la dégradation).

\subsection{Analyse des classes princi- pales de composés organiques}

\subsubsection{Secteur amont, de Saut- Mouche à BPS}

Les teneurs en hydrocarbures aromatiques issus des produits provenant du lessivage des sols ou de l'humification du matériel déposé (Bracewell et Robertson, 1981) tendent à décroître vers l'aval, en surface, signe d'une plus grande fraîcheur du matériel organique (Seres-Aspax et al., 1985), notamment au niveau de la station Tigre avec une légère augmentation sur BPS où le matériel paraît plus dégradé (fig. 9A). 
A

Aval

Phenols totaux
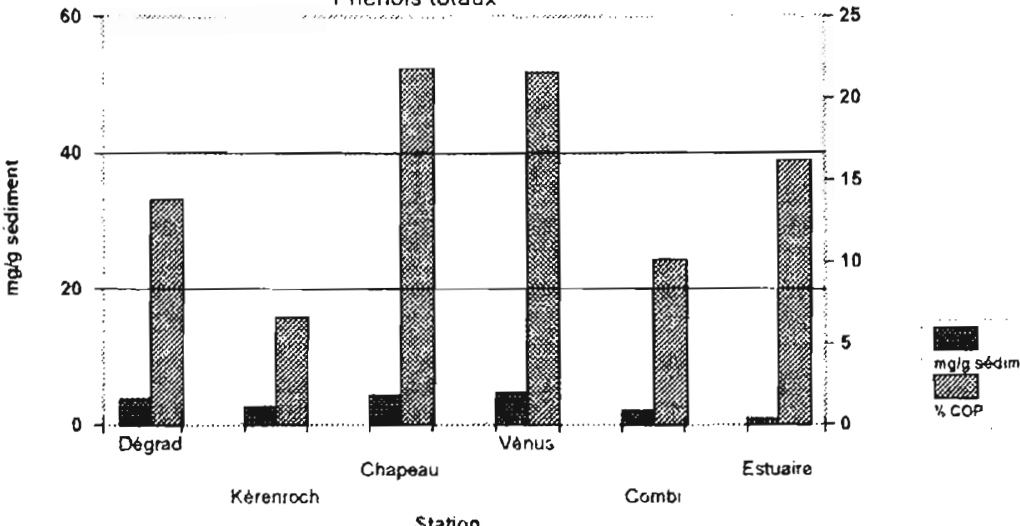

$\%$ COP

B

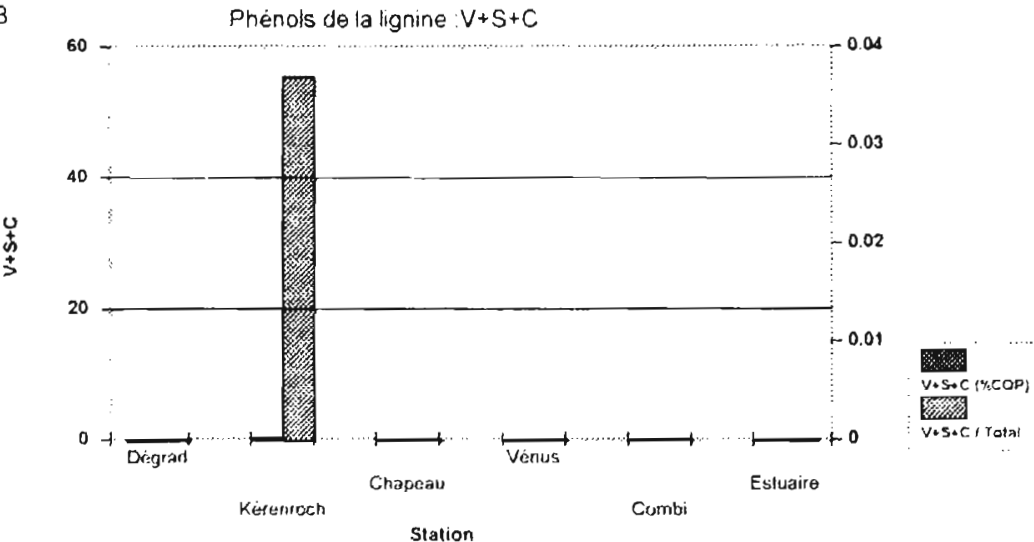

C
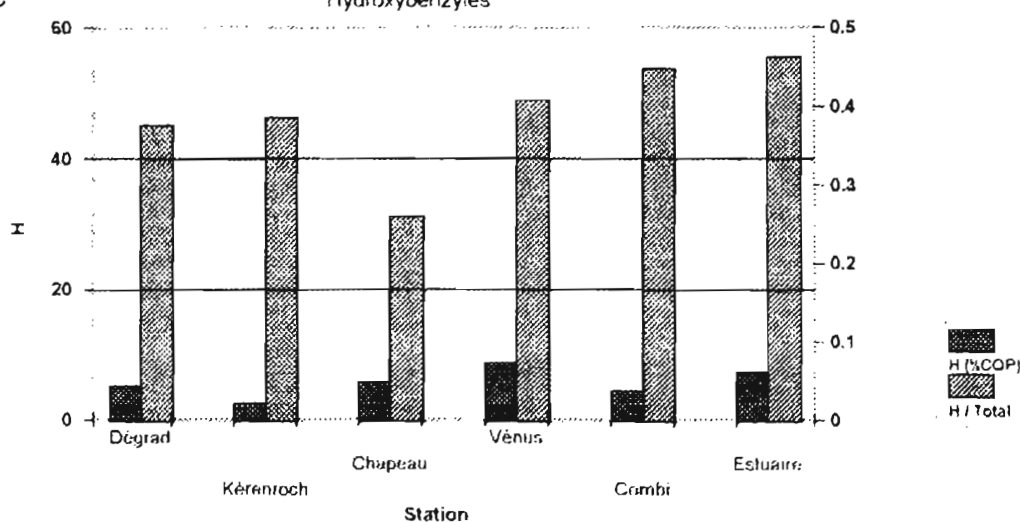

Hi lotal

Station

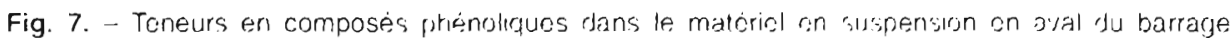
(le Pctit-Saut. A: Teneurs totales en phénols (mg/g-\%, COP). B : composes phéneliques dérivés de la lignine cest-a-dire vanillyles. syringyles ot cinnamyles (V.S+C) ( $\%$ COP. $\%$, phénols totaux). C: composes hydroxybenzyles ( $1 \%$ COP- $-2 \%$ phenols totaux).

Fig. 7. - Contents in phenolic compounds in total suspended matcrial downstream the Petit-Saut dam. A : Total Phenol contents (mg/g-\%, COP). B : Lignin-derived phenolic compounds i.e. Vanillyl. Syringyl and Cinnamyl contents $(V+S+C)(\%$, POC- $\%$ istal phenols). C. Hydroxybenzyl compounds (" $"$, POC-" $"$, istal phenols). 
A
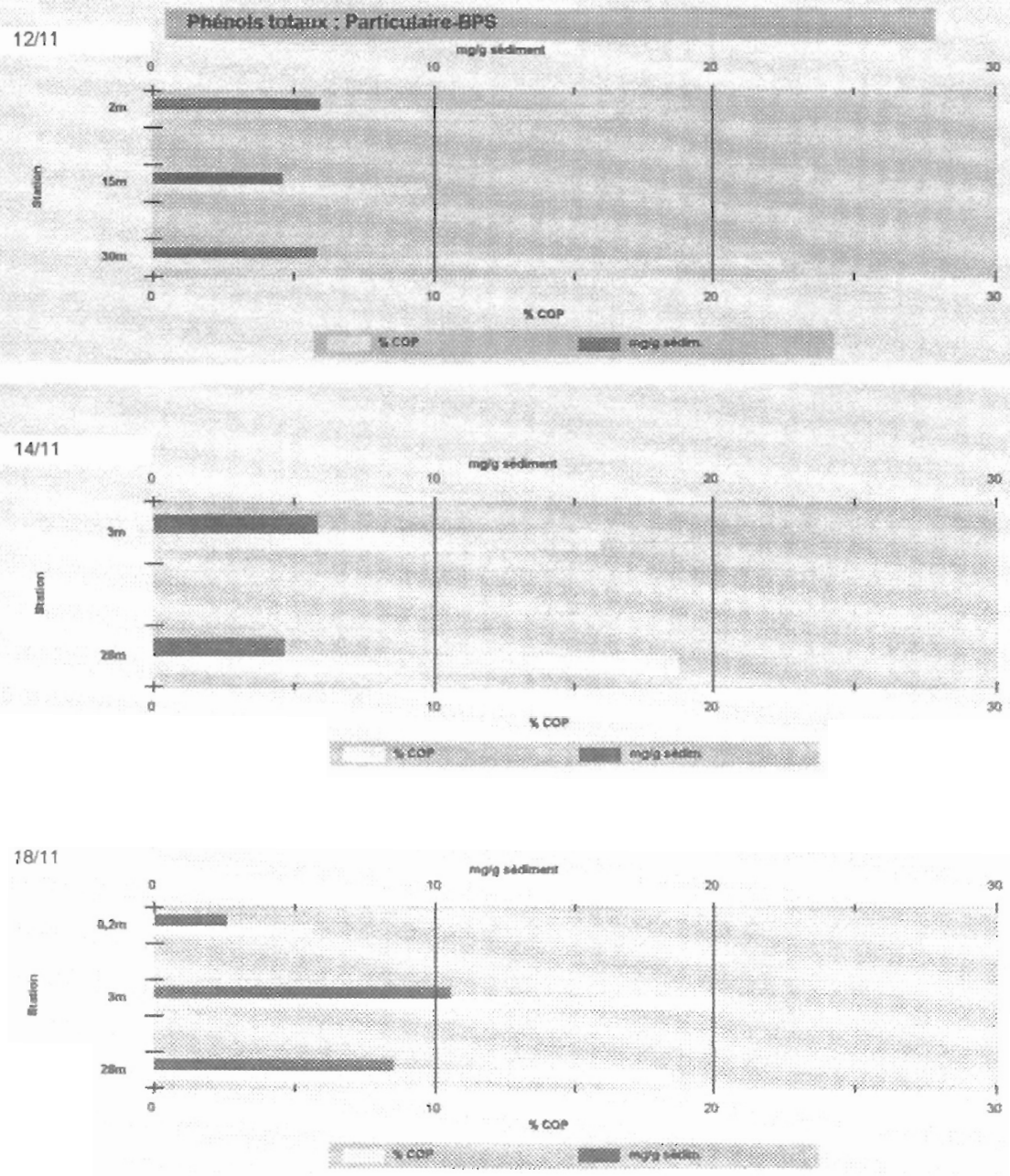

B

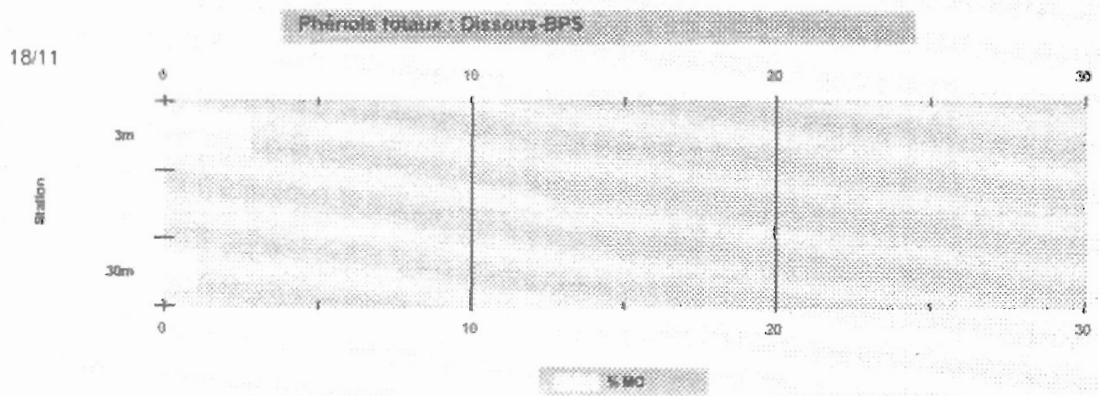

Fig. 8. - Teneurs en composés phénoliques dans le matériel en suspension et le matériel dissous sur des profils verticaux à la station BPS (mg/g \% COP). A : matériel particulaire, prélèvements du 12/11/94, 14/11/94́ et du 18/11/94; B: matériel dissous, prélèvements du 18/11/94. (\% M.O) Fig. 8. - Contents in phenolic compounds in suspended and dissolved material along vertical profiles at BPS (mg/g \% POC). A: particulate material sampling on 12/11/94, 14/11/94 and 18/11/94; B: dissolved material sampling on $18 / 11 / 94$. (\% O.M) 


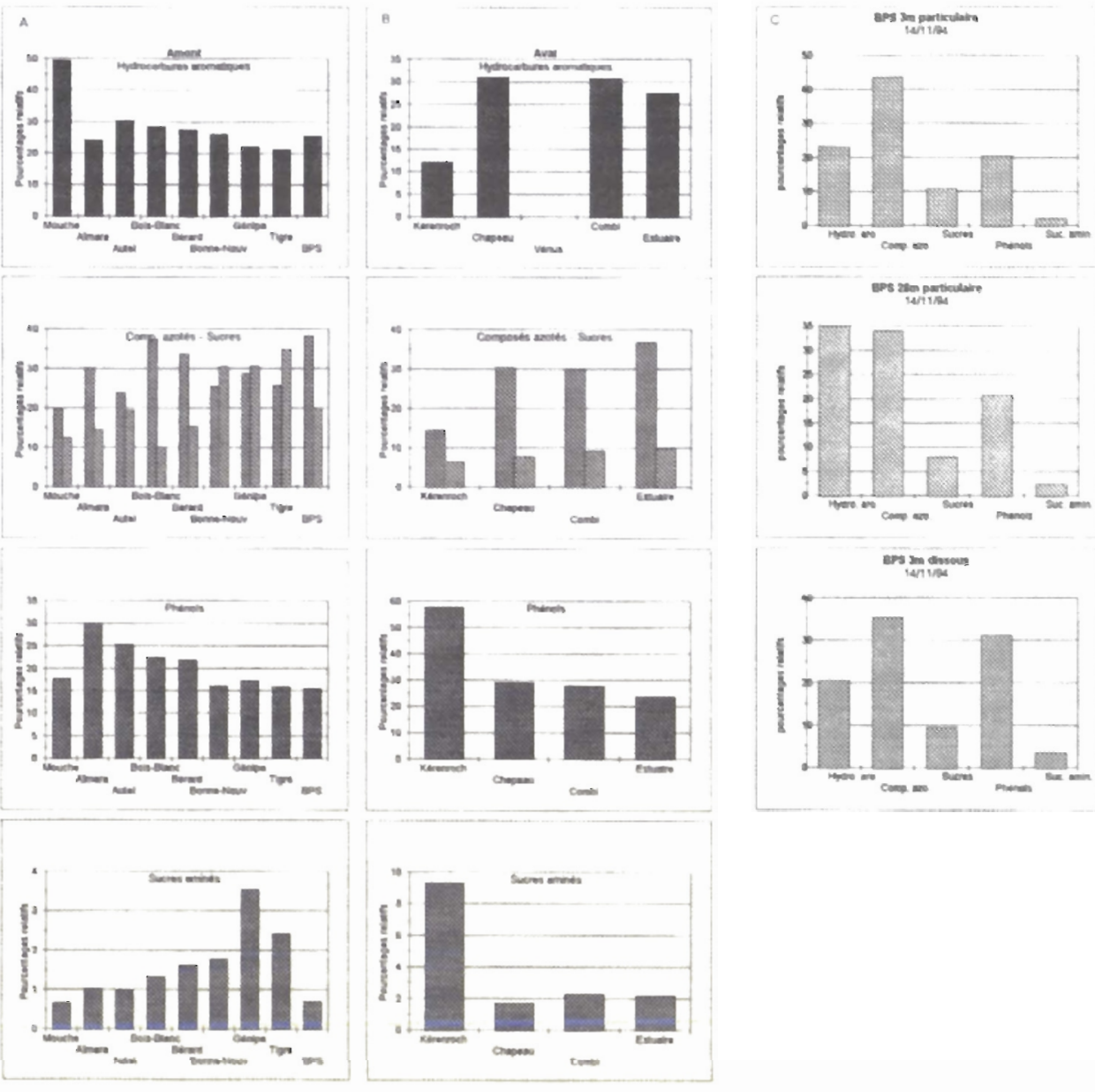

Fig. 9. - Classes majeures de composés organiques dans le matériel en suspension en amont et en aval du barrage de Petit-Saut. Distribution des classes majeures de composés organiques à la station BPS le long de profils verticaux le 14/11/94 (Pourcentages relatifs. Analyse par Py-CG-SM). Fig. 9. - Main classes of organic compounds in total suspended material upstream and downstream the Petit-Saut dam. Distribution of main classes of organic compounds at BPS station along vertical profiles sampled on 14/11/94 (Relative percents. Py-GC-MS analysis).

Les composés azotés subissent des fluctuations avec des valeurs plus fortes sur Bois-Blanc, Bérard et BPS (phytoplancton) (fig. 9A). La plus faible valeur est observée sur Mouche en amont, ce qui paraît normal (lessivage des sols), car la teneur en chlorophylle a est la plus faible (A. Vaquer, 1997). 
Les sucres présentent les teneurs les plus fortes sur les stations BonneNouvelle, Génipa et Tigre, certainement en rapport avec la plus grande abondance du phytoplancton. Cette distribution s'accorde avec celle de la chlorophylle a (fig. 9A).

Les phénols décroissent vers l'aval signe d'une participation moindre des végétaux supérieurs (fig. 9A). Toutefois, ces composés dérivés de la pyrolyse peuvent également provenir des protéines ou de la cyclisation des sucres (Sigléo et al., 1982).

Les sucres aminés suivent la distribution des sucres et soulignent la plus grande abondance du phytoplancton sur Génipa et Tigre qui s'accorde tout-à-fait avec la distribution de la chlorophylle a (fig. 9A).

\subsubsection{Secteur aval, de Dégrad à Estuaire}

Les teneurs en hydrocarbures aromatiques s'accroissent de Kérenroch à Chapeau (signe de dégradation) avec des valeurs plus constantes ensuite (fig. 9B).

Les composés azotés et les sucres s'accroissent vers l'aval au niveau de l'estuaire, comme la chlorophylle a (A. Vaquer, 1997), en rapport avec l'accroissement du caractère marin (fig. 9B).

Les composés phénoliques en toute logique décroissent vers l'aval, ce qui traduit une participation plus marine avec réduction du matériel ligneux (fig. 9B). La station Kérenroch semble se distinguer par des teneurs fortes en phénols et en sucres aminés assez difficilement explicables.

\subsubsection{Station BPS à plusieurs ni- veaux et sur plusieurs jours}

Dans la matière organique particulaire les teneurs en hydrocarbures aromatiques s'accroissent vers la profondeur, (fig. 9C). Au contraire les sucres et les composés azotés, substances biodégradables, décroissent en importance, signe de dégradation dans la colonne d'eau. Les phénols paraissent constants et les sucres aminés sont plus abondants.

Dans le matériel dissous, à $3 \mathrm{~m}$ de profondeur, les teneurs en hydrocarbures aromatiques sont plus faibles que dans le matériel particulaire. Les phénols, au contraire, tendent à s'accroitre (fig. 9C).

La matière organique dans le matériel dissous paraît plus dégradée à $28 \mathrm{~m}$ qu'à $3 \mathrm{~m}$ (plus grande abondance d'hydrocarbures aromatiques et baisse des substances biodégradables).

Les rapports caractéristiques calculés montrent la plus grande fraîcheur du matériel vers l'aval sur Bonne-Nouvelle, Génipa et Tigre (rapport furfural/pyrrol assez haut) et le caractère au contraire très évolué de la matière organique amenée par le fleuve à Mouche (valeur très faible de ce rapport), associé à des hydrocarbures aromatiques très abondants, à des sucres aminés très faibles et à un rapport benzène/toluène très haut, signe de la condensation des noyaux aromatiques dans le matériel (Trasar-Cepeda et al., 1994; fig. 10). D'autre 
part, les stations en aval du barrage présentent un rapport furfural/pyrrol assez faible, signe d'une matière organique évoluée.

Le rapport acétonitrile/pyrrol est fort sur BPS et Chapeau montrant par là la prédominance des composés azotés aliphatiques comparativement aux composés hétérocycliques (fig. 10).

\section{CONCLUSION}

Les faits majeurs observés dans ce domaine fluviatile sont de divers ordres:

Dans le secteur tout-à-fait en amont du barrage, la charge organique d'abord assez réduite, s'accroît jusqu'à BPS alors que, d'un point de vue qualitatif, les apports fluviatiles directs se traduisent par un enrichissement en hydrocarbures aromatiques et en phénols issus du lessivage des sols (matériel ligneux), un rapport furfural/pyrrol bas, un rapport benzène/toluène élevé, associès à une forte baisse de la chlorophyllea (A. Vaquer, 1997).

Un enrichissement en matière organique se produit vers l'aval au niveau des stations Bonne-Nouvelle, Génipa et Tigre. L'accroissement concomitant des sucres, des polysaccharides ef des sucres aminés témoignerait d'une matière organique fraîche provenant du développement du phytoplancton, comme le montrent les valeurs fortes de la chlorophylle a (A. Vaquer, 1997).

A l'aval du barrage, un accroissement de la charge organique intervient au niveau de l'estuaire. Les teneurs en sucres et en phénols décroissent vers l'amont en même temps que les taux de composés azotés s'accroissent, témoignant de l'accentuation du caractere marin. Dans
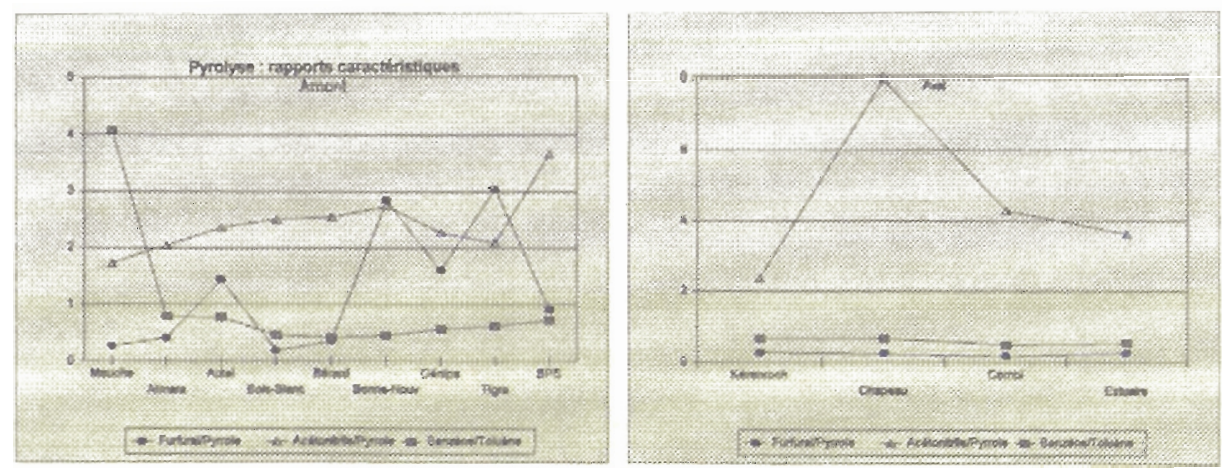

Fig. 10. - Analyse par Py-CG-SM: rapports caractéristiques dans le matériel en suspension, en amont et en aval du barrage de Petit-Saut (furfuralpyrrole, benzène/toluène, acétonitrile/pyrrole). Fig. 10. - Py-GC-MS Analysis : characteristic ratios in total suspended material upstream and downstream the Petit-Saut dam (furfural/pyrrol, benzene/toluene, acetonitrile/pyrrol). 
ce secteur aval, les polysaccharides sont moins abondants et le rapport furfural/pyrrol moins élevé que dans le secteur amont du barrage. Par contre, les teneurs en phénols rapportés au carbone organique sont beaucoup plus fortes, signe d'un matériel plus dégradé, appauvri en sucres et enrichi en composés phénoliques résistants qui s'expliquerait par l'origine des eaux issues des couches profondes du barrage.

Sur les profils verticaux réalisés sur la station BPS, la matière organique est moins abondante dans les couches profondes désoxygénées. Les sucres et les composés azotés décroissent également, signe du développement du phytoplancton en surface et d'une forte dégradation dans la colonne d'eau.

Dans la matière organique dissoute on remarque les indices d'un plus grand état de fraicheur, souligné par la plus grande abondance des sucres aminés et la baisse des hydrocarbures aromatiques.

En terme de biodégradabilité de la matière organique, l'abondance des hydrocarbures aromatiques et, quelquefois, des phénols, un rapport furfural/pyrrol bas (Wilson et al., 1982) et un rapport benzène/toluène élevé témoignent d'un matériel essentiellement détritique, pédogène, dégradé comme sur la station Saut-Mouche tout-à-fait en amont. Vers l'aval au contraire, le matériel, plus frais, où la composante aquogène prend de plus en plus d'importance, est surtout enrichi en sucres et quelquefois en composés azotés. Un rapport furfu- ral/pyrrol élevé témoigne d'ailleurs bien de la fraîcheur de ce matériel aux stations Tigre et Bonne-Nouvelle et s'accorde assez bien avec la distribution de la chlorophylle a (A. Vaquer, 1997).

En aval du barrage, divers caractères montrent que la matière organique est plus dégradée qu'en amont (décroissance des sucres, enrichissement en phénols résistants), ce qui s'expliquerait par l'origine des eaux qui proviennent de lachers par ouverture des vannes de fond du barrage.

Les analyses réalisées au cours de cette mission permettent donc de mettre l'accent sur trois points principaux :

1. I'hétérogénéité des sources le long du plan d'eau : eaux fluviatiles en amont du barrage chargées en matériel issu du lessivage des sols, apports des affluents, développement du phytoplancton vers l'aval, abondance des feuilles.

2. le rôle du barrage et de son fonctionnement: les sucres et d'autres composés biodégradables décroissent en aval du barrage. Bien que les teneurs en phénols rapportées au carbone organique s'accroissent, les composés pédogènes comme les phénols de la lignine ont tendance à diminuer au sein de la matière organique après la retenue. Parmi ces composés phénoliques les hydroxybenzyles se maintiennent à des niveaux élevés et seraient en grande partie d'origine phytoplanctonique ou foliaire. Le matériel organique provenant des couches d'eaux profondes du barrage paraît donc plus 
évolué (baisse des sucres et du rapport furfural/pyrrol et augmentation des hydrocarbures aromatiques). Le caractère marin s'accentue en aval du barrage et se traduit par un accroissement des composés azotés et des sucres.

L'effet de turbinage entraîne une perte de matière organique et une forte dégradation de celle-ci.

Le caractère partiellement hypothétique des conclusions que nous avons pu tirer de nos résultats tient principalement au fait que les prélèvements sont effectués le long d'un transect longitudinal unique, échelonnés sur deux jours. La position des points de prélèvement par rapport aux berges et au milieu du courant ont une incidence que nous n'avons pu apprécier. La date des prélèvements par rapport au cycle annuel du débit du Sinnamary est susceptible d'amoindrir le caractère général des conclusions qui sont corroborées, soulignons-le, par les résultats des analyses des pigments chlorophylliens de A. Vaquer, souvent cité.

\section{REMERCIEMENTS}

Ce travail a été réalisé grâce au support financier des services d'EDF - CNEH que nous remercions ainsi que nos collègues catalans de l'Institut Quimic de Sarrià pour l'aide qu'ils nous ont apportée dans la réalisation des analyses de pyrolyse.

\section{RÉFÉRENCES BIBLIOGRAPHIQUES}

Alekin O.A., 1978. Water erosion of land surface. USSR Committee for the Int. Hydrol. Decade. Studies and reports in Hydrology $n^{\circ} 25$ - Unesco.

Bracewell J.M. et Robertson, 1981. Analytical potential of pyrolysis products derived from soil organic matter. Analytical Proceedinds, $18: 532-535$.

Buffle J., Zali O., Zumstein J. et De Vitre R., 1987. Analytical methods for the direct determination of organic and inorganic species: seasonal changes of iron, sulfur and pedogenic and aquogenic organic constituents in the eutrophic lake Bret, Switzerland. Sci. Total Environ., 64 : 41-59.

Charrière B., Sancho L., Serve L., Combaut G., Gadel F. et Piovetti L., 1986. Les composés phénoliques dans un écosystème lagunaire méditerranéen: végétaux aquatiques, eaux, sédiments. Journées Internationales d'Etudes du Groupe Polyphénols, Montpellier 13 : 560-564.

Gadel F., Charrière B., Serve L. et Comellas L., 1992. Caractérisation chimique des composés humiques et de leurs diverses classes de poids moléculaires dans les dépōts du delta du Rhône. Oceanol. Acta, 15, 1: 61-74.

Gallali T., 1972. Etude statique et dynamique des polysaccharides et des aminopolysaccharides au cours de l'humification. Thèse Université Nancy, $93 \mathrm{p}$.

Garrels R.M., Mackensie F.T. et Hunt C., 1973. Chemical cycles and the global environment. Los Altos Calif., W. Kaufmann inc., $206 \mathrm{p}$.

Hartley R.D., 1971. - Improved method for the estimation by gas-liquid chromatography of lignin degradation from plants. - J. Chromatogr., 54, 335-344.

Hartley R.D. \& Buchan H., 1979. - Highperformance liquid chromatography of phenolic acids and aldehydes derived 
from plants or from the decomposition of organic matter in soil. - J. Chromatogr., $180: 139-143$.

Hedges J.I. \& Parker P.L., 1976. - Landderived organic matter in surface sediments from the Gulf of Mexico. Geochim. Cosmochim. Acta, 40 : 1019-1029.

Hedges J.I. \& Mann D.C., 1979. The characterization of plant tissues by their lignin oxidation products. - Geochim. Cosmochim. Acta, 43: 1809-1818.

Hedges J.I. \& Ertel J.R., 1982. Characterization of lignin by gas capillary chromatography of cupric oxide oxidation products. - Anal. Chem., 54 : 174-178.

Hedges J.I., Clark W.A., Quay P.D., Richey J.E., Devol A.H. \& Santos U.de M., 1986. - Compositions and fluxes of particulate organic material in the Amazon River. - Limnol. Oceanogr., 31 (4), 717-738.

Ibrahim R.K., Towers G.H.N. \& Gibbs R.D., 1962. - Syringic and sinapic acids as indicators of differences between major groups of vascular plants. - J. Linn. Soc. (Bot.), 58 (372) : 223-230.

Livingstone D.A., 1963. Chemical composition of rivers and lakes. Data of Geochemistry. Us Geological Survey Prof. Paper 440-G, 64 p.

Martin J.M. et Meybeck M., 1979. Elemental mass-balance of material carried by world major rivers. Mar. Chem., 7 : 173-206.

Meybeck M., 1982. Carbon, nitrogen and phosphorus transport by world rivers. Am. J. Sci., 282 : 401-450.
Pocklington R. \& MacGregor c.d., 1973. - The determination of lignin in marine sediments and particulate form seawater. - Intern. J. Environ. Anal. Chem., $3: 81-93$.

Seres-Aspax A., Alcañiz-Baldellou J.M. et Gassiot M. 1985. Application of pyrolysis-gas chromatography to the study of the composting process of barley straw and pear-tree wood. J. Anal. Appli. Pyrol., 8 : 415-426.

Sigleo A.C., Hoering T.C. et Helz G.R., 1982. Composition of estuarine colloidal material: organic components. Geochim. Cosmochim. Acta, 46 : 1619-1626.

Trasar-Cepeda M.C., Ceccanti B., Leiros M.C., Calcinai M. et Gil-Sotres F., 1994. Characterization of organic matter in lignite minesoils at various ages by pyrolysis-gas chromatography. In Humic Substances in the Global Environment and Implications on Human Health. N. Senesi and T.M. Miano, ed. : 707-712.

Vaquer A., 1997. Distribution spatio-temporelle du phytoplancton dans le réservoir de Petit-Saut (Guyane française). Hydroécol. Appl. 9 (1/2) : pp. 169-193.

Wilson M.A., Philp R.P., Gillam A.H., Gilbert T.D. et Tate K.R., 1982. Comparison of the structures of humic substances from aquatic and terrestrial sources by pyrolysis-gas chromatography-mass spectrometry. Geochim. Cosmochim. Acta, 47 : 497-502. 